\title{
Hemorragia Subaracnóidea Espontânea devida a Aneurismas Cerebrais
}

\author{
Mirto N. Prandini*
}

\section{RESUMO}

A hemorragia subaracnóidea espontânea ainda se constitui em sério problema médico com altos indices de mortalidade e morbidade. A ruptura de aneurisma cerebral corresponde a cerca de $80 \%$ dos casos de hemorragia subaracnóidea espontânea nos adultos e em $55 \%$ dos casos na infáncia. $O$ ressangramento e o vasoespasmo săo as causas principais de mortalidade. Atualmente, novas drogas estão sendo desenvolvidas para o combate ao vasoespasmo. A hipotermia moderada tem provado ser o melhor agente protetor cerebral. Com novas técnicas de abordagem, novos materiais para clips e exames mais sofisticados, o prognóstico tem melhorado consideraveimente. Alguns tópicos gerais sāo discutidos.

\section{UNITERMOS}

Hemorragia subaracnóidea; hemorragia cerebral; aneurismas cerebrais.

\section{INTRODUÇÃO}

A hemorragia subaracnóidea espontânea, independente do tratamento cirúrgico instituído, constitui-se em entidade cuja abordagem não se restringe apenas ao neurocirurgião mas ao neurologista, clínico geral, ginecologista, etc., que vão participar permanentemente do atendimento ao paciente e cujas dificuldades, inerentes à própria doença, sāo igualmente divididas. Alguns dos aspectos gerais mais comuns serão aqui abordados e analisados sob o ponto de vista do momento atual e que felizmente vem se modificando com bastante velocidade.

\section{DEFINIÇÃO}

Hemorragia subaracnóidea (HSA) ou meníngea é o extravasamento de sangue no interior do espaço subaracnóide, proveniente da ruptura de vasos aí contidos. Nesse espaço está contido o líquido cefalorracjueano, que se torna hemorrágico.

\section{ETIOLOGIA}

A HSA, de forma mais geral, tem como causas mais freqüentes o aneurisma (An) e a malformaçāo arteriovenosa $(60 \%)$. A hipertensão arterial, o sangramento de tumores cerebrais, as discrasias sangüíneas, o uso de anticoagulantes sāo outras causas, mais raras. Devemos lembrar que em $10 \%$ dos casos não se consegue detectar a origem da HSA. Nessas circunstâncias, o exame angiográfico deve ser repetido após um perído de três a oito semanas, quando se poderá mais seguramente considerar como resultado negativo, ou seja, ausência de patologia detectável ao exame.
Professor Adjunto da Disciplina de Neurocirurgia. Escola Paulista de MedicIna. Universidade Federal de São Paulo. 


\section{DLAGNÓSTICO}

Atualmente, o primeiro exame indicado é a tomografia computadorizada (TC) que, até o décimo dia pós-hemorragia, costuma mostrar a presença de sangue nas cisternas encefálicas, sulcos corticais ou até nos ventrículos, confirmando o diagnóstico. A TC pode também demonstrar a presença de hematoma intracerebral, intraventricular, edema cerebral, dilatação do sistema ventricular e mesmo do aneurisma, quando maior que $0,8 \mathrm{~cm}$. Atualmente, dispomos da tomografia helicoidal ou em espiral que nos apresenta imagens mais nítidas das estruturas, inclusive dos vasos intracranianos, com a vantagem de uma duração bastante reduzida do exame. A angiografia por TC (CTA) permite a detecção de An com tamanho de até $2,5 \mathrm{~cm}$ com uma precisão de $95 \%$. Sua indicação máxima reside nos casos de grande urgência cirúrgica pela presença de hematoma, em que a angiografia poderia retardar a intervenção cirúrgica por um período incompatível. Desse modo, a TC pode ser bastante útil tanto no diagnóstico da hemorragia como na indicação do local do sangramento, o que vai orientar o cirurgião no planejamento de sua abordagem ${ }^{13}$. Consideramos contra-indicada a punção lombar para diagnóstico, a não ser quando a TC não confirma a presença de sangue.

\section{INCIDÊNCIA}

Com os novos métodos diagnósticos e a maior facilidade na execução dos mesmos, tem sido diagnosticado um número cada vez maior de HSA por An. Os dados apontam para uma incidência de 10 a 12 HSA por An por ano para cada 100.000 habitantes, ou seja, para uma cidade pequena de 100.000 habitantes deve haver no mínimo uma cirurgia por mês. As mulheres apresentam duas vezes mais An, quando único. No caso de An duplo, a proporção sobe para $3 / 1$ e, no caso de 3 ou mais An, a proporção chega a 11 mulheres para cada homem. Os An são múltiplos em cerca de $27 \%$ dos casos. O território vertebrobasilar corresponde a aproximadamente 5 a $10 \%$ dos casos, sendo os demais pertencentes ao território carotídeo. A artéria corótida interna, se considerada em todos os seus segmentos, é o vaso onde incidem a maioria dos $\mathrm{An}^{9,14}$.

\section{CLÍNICA}

A cefaléia de instalação súbita, de forte intensidade, holocraniana, podendo ser irradiada para o dorso é o sintoma mais constante. Permanece por alguns dias e pouco se modifica com os analgésicos comuns. De início, além da cefaléia, o paciente pode apresentar vômitos, intenso mal-estar geral, tonturas, distúrbios vegetativos com intensa palidez cutânea, podendo ocorrer alterações da consciência; a pressão arterial pode estar elevada pelas próprias condições da afecção, sendo muitas vezes erroneamente interpretada como crise hipertensiva simples e medicada como tal. A partir desse momento, o paciente necessita de cuidados extremos pela delicadeza do quadro que é bastante instável, podendo evoluir rapidamente para o óbito.

Os aneurismas cerebrais são responsáveis pela HSA espontânea em $80 \%$ dos casos nos adultos e em $55 \%$ nas crianças. Os aneurismas são malformações saculares das paredes arteriais, que ocorrem devido a uma deficiência congênita da parede vascular (túnica média) associada a uma degeneração progressiva da camada elástica, produzindo invaginação progressiva da íntima através dessas partes mais fracas. Essas alteraçōes são mais encontradas ao nível da bifurcação dos maiores vasos intracranianos junto à base do encéfalo. Nesses pontos de bifurcação é onde ocorre o maior impacto da pressão sangüínea. Vários fatores têm sido sugeridos no sentido de se ligar a presença de aneurismas a outros demais elementos. Deficiência de colágeno tipo III nas paredes arteriais tem sido encontrada significativamente nos casos de aneurismas rotos ${ }^{22}$. A biópsia de pele para a detecção dessa deficiência foi positiva em casos familiares com aneurismas rotos e não-rotos, sendo sugerido como um dos exames de utilidade na indicação de estudos complementares em parentes de pacientes com aneurismas que sangraram. A associação com outras doenças do colágeno (síndrome de Marfan, pseudoxantoma elástico e síndrome de Ehlers-Danlos, tipo IV) já foi provada. Outro elemento que tem sido estudado é a elevação dos níveis séricos da lipoproteína A (Lp A). Essa elevação é mais constante em mulheres jovens com aneurismas rotos ${ }^{3}$. Pode-se facilmente associar a existência de An com vasculopatias. De fato, a incidência três vezes maior de coronariopatias já foi assinalada há muitos anos em pacientes com HSA por An. A associação com hipertensão arterial está presente em cerca de $27 \%$.

A HSA por aneurisma ocorre mais freqüentemente entre os 30 e 50 anos de idade. Os An podem ter tamanhos diferentes, variando de mínimo $(2 \mathrm{~mm})$ até gigantes (acima de 2,5 cm). Costuma-se dividi-los em saculares (até $1,5 \mathrm{~cm}$ ), globosos (de 1,5 a $2,5 \mathrm{~cm}$ ) e gigantes (com diâmetro acima de $2,5 \mathrm{~cm}$ ). Do ponto de vista cirúrgico, os saculares e gigantes têm comportamento bastante semelhante, embora os gigantes, por apresentarem uma 
parede espessa formada por coágulos organizados, mais raramente levam à HSA. No entanto, podem apresentarse com sinais localizatórios, comprimindo nervos cranianos, etc. Os An da porção supraclinóidea da artéria carótida ou da artéria comunicante posterior também podem dar como sinal localizatório a paralisia súbita ou rapidamente progressiva do nervo oculomotor (ptose, desvio do globo ocular para fora e midríase), com ou sem a presença de HSA. Os aneurismas localizados dentro do seio cavernoso, infraclinóideos, são em geral grandes, gigantes, não costumam romper e sua sintomatologia está relacionada com a paralisia dos nervos oculomotores e dor ocular.

\section{CONDUTA}

Uma vez feito o diagnóstico da HSA, o paciente deve ser submetido ao estudo angiográfico dos 2 sistemas vasculares: carótidas e vertebrobasilar, mesmo que haja sinais localizatórios devido à presença de An múltiplos. Quando há An múltiplos, a TC pode ajudar no sentido de orientar qual o aneurisma responsável pelo sangramento. Nesses casos, a presença de um pequeno ou grande hematoma, uma cisterna lateral mais cheia de sangue, são indícios do aneurisma que rompeu. Para o cirurgião, tal informação é de vital importância, pois a cirurgia efetuada em local não-correspondente ao aneurisma que sangrou costuma ter resultados bastante sombrios. Em cerca de 10\% dos casos, o estudo angiográfico pode não demonstrar a existência de anormalidades. A conduta mais difundida é a repetição do exame em um prazo de duas a oito semanas. Persistindo o resultado negativo, o paciente é mantido em observação clínica. Alguns autores advogam a exploração cirúrgica mesmo nessas circunstâncias, em caso de a TC ser bastante sugestiva de sangramento ou por meio da ressonância magnética, que é um exame mais apurado para a determinação da presença de sangue em suas várias fases de degradação. No caso de confirmação do mesmo em locais definidos, microaneurismas podem ser encontrados em cerca de $40 \%$ dos casos e convenientemente protegidos.

\section{COMPLICAÇÕES}

Três complicaçōes são freqüentes: o hematoma intracerebral, o ressangramento e o vasoespasmo.

O hematoma intracerebral ocorre no momento do sangramento e é responsável pelo maior comprometimento da consciência, presença de sinais localizatórios e maior mortalidade. Produzindo efeito de massa, se não tratado adequadamente, aumenta a mortalidade e a morbidade ${ }^{25.27}$.

$O$ ressangramento é complicação frequiente e ocorre mais comumente nas primeiras 24 horas, mas até o $10^{\circ}$ dia sıa frequiência é grande. Dados recentes de estudos cooperativos demonstram a presença de ressangramento em cerca de $33 \%$ dos pacientes durante os primeiros trinta dias. Considerando-se esses casos isoladamente, observamos que até o segundo dia ocorrem $20 \%$ dos ressangramentos; um novo pico na incidência será observado a partir do oitavo dia, havendo um expressivo declínio após o décimo quarto dia. Se na primeira HSA morrem cerca de $40 \%$ dos pacientes, na segunda essa porcentagem sobe para mais de $60 \%{ }^{27}$.

O vasoespasmo ocorre devido à HSA, onde há liberação de substâncias vasoativas com mecanismos ainda não totalmente conhecidos. Sua instalação é mais comum após o 3, 4 dia da HSA, mas pode ocorrer até 1 ou 2 semanas após. Sua presença é suspeitada quando há piora do estado de consciência e instalação de sinais localizatórios. Seu aparecimento tem relação com o maior enchimento de sangue das cisternas da base. Pode também ocorrer depois da cirurgia, sendo o elemento mais temido pelo cirurgião pois é a maior causa de óbitos ou secjüelas pós-operatórias.

Outra complicação é a obstrução da circulação do LCR levando à hidrocefalia interna, que pode ser de instalação subaguda ou crônica. A hidrocefalia leva à hipertensão intracraniana em cerca de $50 \%$ dos casos, requerendo, pois, derivação liquórica com válvula nesses casos. Havendo hidrocefalia interna sintomática, o paciente fica apático, sonolento, com tendência à piora.

\section{TRATAMENTO}

O paciente com HSA deve ser internado e receber cuidados especiais. O repouso absoluto no leito é necessário, o estresse deve ser evitado com o uso de benzodiazepínicos, o combate à dor, com analgésicos. Qualquer esforço físico deve ser evitado. O aumento inicial da pressão arterial deve ser combatido com o uso de diuréticos, hipotensores, porém mantendo-a em níveis pouco acima dos normais para o paciente. Para a cefaléia intensa usamos glicose hipertônica, $40 \mathrm{ml}$ a $50 \%$, associada a analgésicos potentes.

O uso de corticóides (dexametasona - $20 \mathrm{mg}$ diários) ajuda na recuperação do paciente. $\mathrm{O}$ uso de substânciass hiperosmóticas como manitol $25 \%, 100 \mathrm{ml}$ ciadal 4 ou 6 horas, está indicado nos casos com hipertensão intracraniana mais acentuada. A sondagem vesical, 
sondagem gástrica, intubação endotraqueal, controle da pressão arterial média, da pressão venosa central, são reservados para os casos mais graves.

Substâncias antifibrinolíticas (ácido epsilon aminocapróico) não têm sido mais usadas em nosso meio. Sua administração reduz o ressangramento, porém as taxas de hidrocefalia chegam a duplicar enquanto que a isquemia sintomática aumenta significativamente. A nimodipina, quando administrada logo de início, reduz o vasoespasmo em até $30 \%$ dos casos.

Quando da admissão no hospital, a determinação do estado de consciência é muito importante. Consideramos 5 graus, de acordo com a classificação de Botterell: grau 1 - paciente plenamente consciente; grau 2 - paciente sonolento, com ou sem sinais meníngeos; grau 3 torporoso, com ou sem sinais neurológicos focais; grau 4 - coma superficial; grau 5 - coma profundo. Os pacientes nos 2 primeiros graus terão o parênquima cerebral em melhores condições. Nesses casos, hoje em dia, está indicado o estudo angiográfico imediato seguindo-se da cirurgia do aneurisma. É a chamada cirurgia ultraprecoce ou precoce, feita até 72 horas após a hemorragia. Com isso, evita-se o ressangramento, permitindo uma limpeza do sangue contido nas cisternas da base cuja presença facilita o aparecimento do vasoespasmo. A cirurgia permite ainda que a pressão arterial e o volume circulante sejam aumentados, evitando a instalação do vasoespasmo. Em alguns serviços, as cirurgias continuam sendo feitas de 7 a 14 dias após o sangramento. Nesses casos, os resultados cirúrgicos podem ser melhores, mas o risco de ressangramento também é maior nesse período em que o paciente está aguardando a cirurgia.

Nos dias atuais, com as técnicas de microcirurgia, a mortalidade e a morbidade para os casos de aneurisma baixaram a níveis alentadores (5 a 10\%), se considerarmos os pacientes até o grau 3.

Quando a TC mostra a presença de um hematoma intracerebral produzindo efeito de massa, a cirurgia deve ser imediata ${ }^{14.27}$. O hematoma deve ser evacuado e, se possível, o aneurisma clipado, sendo portanto interessante a feitura do estudo angiográfico previamente, uma vez que o estado neurológico permita-o. A mortalidade nos graus 4 e 5 é altíssima. O paciente em grau 3 tanto pode melhorar passando para graus 2 e 1 , como pode piorar. Nesses três últimos graus, quando não há outra causa além da HSA que possa estar influindo (hematoma, hidrocefalia), cotumamos aguardar pela melhora do paciente para operá-lo. O tratamento cirúrgico "definitivo" consiste na clipagem do colo do aneurisma. No pós-operatório são importantes os cuidados clínicos gerais e especiais. As cirurgias dos aneurismas devem ser feitas em centros bem adaptados, com anestesistas e neurocirurgiões experientes, microscópio cirúrgico, variedades de clipes, etc. De outra maneira, é mais seguro conduzir o paciente para um centro especializado. A cirurgia em locais inadequados costuma ser catastrófica ${ }^{14,15,24}$.

$O$ vasoespasmo, uma vez instalado, é de difícil combate. A técnica dos $3 \mathrm{H}$ (hipertensão arterial induzida, hemodiluição e hipervolemia) em alguns casos mais leves chega a trazer bons resultados. Pelas suas características, leva a um aumento do risco de ruptura do An e, portanto, só pode ser usada nos casos já operados. A busca de substâncias vasoativas encontrase em fase de grande expansão, com resultados preliminares encorajadores.

As substâncias trombolíticas têm sido tentadas, como o uso do ativador de plasminogênio tissular com administração nas cisternas encefálicas. Em um recente estudo multicêntrico ${ }^{(6)}$, foi constatada a sua eficácia quanto à melhoria do vasoespasmo, mas houve significativo aumento do sangramento dos An nãoclipados. Seu uso, portanto, é indicado nos casos já operados, sem os riscos da ruptura do An .

A angioplastia transluminal (Zubokov, 1984) para casos de vasoespasmo secundário tem sua indicação bastante restrita, pois complicaçōes como ruptura do An no local da clipagem, embolia à distância e transformação do enfarte em hemorrágico foram descritas, aliadas às dificuldades técnicas do método. Embora muito ainda exista a ser desvendado, a contração vascular parece ser de dois tipos: ativa e passiva ${ }^{5}$. A primeira, dependente de mecanismos funcionais, poderia ser revertida com o auxílio de drogas; a segunda, já resultante de alterações estruturais da parede arterial, teria pouca reação aos medicamentos, sendo mais sensível a processos mecânicos (angioplastia endovascular).

Quando há aneurismas múltiplos, todos eles devem ser operados, na mesma cirurgia quando possível ou em cirurgias separadas. Os aneurismas chamados incidentais, descobertos ao acaso, têm indicações cirúrgicas, uma vez que as condições clínicas permitamna. A mortalidade cirúrgica costuma ser extremamente reduzida nesses casos ${ }^{15}$.

\section{Mecanismos de proteção cerebral}

Se os resultados cirúrgicos melhoraram significativamente após a introdução das técnicas microcirúrgicas, com os estudos atuais da proteção cerebral tanto com a hipotermia moderada como com drogas 
neuroprotetoras, alguns casos antes inoperáveis podem ser abordados com mais segurança e com resultados bastante animadores.

Esse capítulo tem sido dos mais estudados na última década. Para um eficiente combate à ação deletéria do sangramento, faz-se necessário um minucioso conhecimento dos elementos envolvidos nos processos de degradação do sangue no espaço subaracnóideo e dos fenômenos que ocorrem no cérebro após um quadro isquêmico'. Certamente, a cada dia, maiores são os conhecimentos adquiridos. Alguns elementos já são conhecidos como participantes do processo e o Ca+t ocupa uma posição destacada pois, embora seja elemento fundamental para ativação celular, sua concentração sendo 10.000 vezes maior no espaço extracelular, o seu ingresso no citoplasma torna-se bastante facilitado em quaisquer alteraçōes que venham a ocorrer, principalmente nos casos de hemorragia, isquemia, convulsões e trauma.

Muito resumidamente poderíamos apontar algumas das açōes do $\mathrm{Ca}++$ no quadro de HSA:

- despolarização da membrana neuronal

- aumento da atividade sináptica

- disfunção das organelas citoplasmáticas

- depleção de energia celular (a célula despende 1 ATP para cada $\mathrm{Ca}++$ que retira de seu interior)

- acidose celular (pela necessidade aumentada de energia e ciclo anaeróbico)

Baseados nesses fenômenos, novas drogas têm sido pesquisadas no sentido de um bloqueio de cada uma das fases acima ${ }^{5,26}$.

Não só o parênquima é afetado; a ação nos vasos cerebrais é importante.

\section{Ação nos vasos cerebrais}

O óxido nítrico (NO) é produzido no endotélio dos vaısos cerebrais ${ }^{28}$, sendo um potente vasodilatador, não possuindo portanto efeito nocivo. Por ação do CA++ intravascular, o NO reage com radicais do oxigênio (superóxido), tranformando-se no peroxidonitrito, elemento altamente tóxico. Uma substância tem sido utilizada com a finalidade de bloquear o excesso de NO (polictilenoglicol-superóxido dismutase), com efeitos apreciáveis.

\section{Neurotransmissores excitatórios}

Os mais importantes são o aspartato e, principalmente, o glutamato, considerado o mais potente neurotransmissor excitatório.
Um dos efeitos do aumento do Ca++ é elevar a excitabilidade celular, havendo um aumento do glutamato $(\mathrm{Gt})$ que se torna elemento tóxico celular. Há evidências também de uma migração do Gt das áreass isquêmicas para as áreas vizinhas (penumbra)', com ação tóxica principalmente às células cujo aporte de $\mathrm{O} 2$ encontra-se reduzido.

Dois são os caminhos procurados: reduzir al neurotransmissão excitatória e reduzir o Gt. Para este último, algumas drogas como o MK-801 e o maleato de dizocalpine, in vitro e em animais, têm apresentado efeitos comprovados. Para a redução da neurotransmissão excitatória, a nizofenona e os hidantoinatos parecem ter ação favorável ${ }^{5,26,28}$.

\section{Hipotermia}

Atualmente é considerado como o elemento de maior proteção cerebral. Com a utilização da hipotermia moderada $\left(33^{\circ} \mathrm{C}\right)$ e regional (no caso apenas cercbral), evitam-se os problemas apresentados classicamente como arritmias cardíacas, acidose metabólica, coagulaçāo intravascular e outras, na fasc pósoperatória, elementos estes que dificultaram muito o seu emprego. A hipotermia, na última década, tem sido cada vez mais estudada, pois as suas vantagens têm sido demonstradas, inclusive em casos de períodos longos de isquemia (acima de uma hora). Com a hipotermia regional, a clipagem temporária do vaso nutriente pode ser efetuada de modo seguro por períodos de tempo matis prolongados. Para cirurgias de aneurismas complexos, tal procedimento técnico pode ser fundamental. A associação de hipotermia, hipertensão moderada (com fenilefrina, que possui pouca ação vasoconstritora cerebral) e manitol ${ }^{20}$, mostrou-se estatisticamente eficiente em um estudo comparativo efetuado ${ }^{7.8 .18 .21}$.

\section{Novas técnicas}

Com as novas abordagens da cirurgia da base de crânio $^{24}$, alguns aneurismas até então inacessíveis puderam ser mais seguramente clipados. Hoje em dia, praticamente qualquer aneurisma pode ser tecnicamente abordado, estando as limitações mais na dependência do estado clínico do paciente, idade, sequielas resultantes da hemorragia, etc. Ainda em casos muito raros, a ligadura da carótida cervical pode ter suas indicações.

A angiografía intra-operatória tem sido vista com muito entusiasmo, principalmente em casos de An complexos. Complicaçōes como estenose de vaso nutriente ou persistência de saco aneurismático foram 
encontradas em cerca de $18 \%$ dos casos selecionados, submetidos ao exame.

A ressonância magnética funcional é um exame dinâmico, pois permite determinar alterações regionais do volume circulatório, fluxo circulatório, oxigenação e metabolismo cerebral e suas variações na dependência da atividade cerebral. A sua utilidade reside na possibilidade da exploraçāo do estado e da topografia funcional, permitindo uma precisa avaliação pré e pósoperatória das áreas em estudo e assim a melhor orientação quanto ao melhor período para a intervenção. Permite também uma melhor avaliação dos resultados cirúrgicos e evolução neurológica.

O desenvolvimento da RM determinou a necessidade de novos clips compatíveis com material nãoferromagnético. Alguns centros importantes estāo usando rotineiramente os clips de titânio, até o momento os que melhor se adaptaram às condiçōes requeridas ${ }^{16}$.

O uso das espiras destacáveis, introduzidas por Guglielmi ${ }^{12}$, constitui-se em um novo capítulo no tratamento dos An. A técnica endovascular ganhal novos adeptos a cada dia; entretanto, os melhores resultados obtidos são justamente nos casos em que a cirurgia convencional encontra as maiores facilidades, com índices baixos de complicações e com a segurança de cura definitiva ${ }^{2}$. Uma nova aplicação da técnica endovascular está se consolidando nos casos em que o paciente dá entrada no hospital sem condições de cirurgia, podendo, entretanto, ser submetido à embolização por espirais nas primeiras horas. Foi alcançado um índice de $69 \%$ de oclusāo total do saco aneurismático". Tal procedimento facilitaria o tratamento mais intensivo do vasoespasmo logo de início e, até a cirurgia convencional, posteriormente, se for o caso.

\section{ACOMPANHAMENTO AMBULATORIAL}

Para os pacientes em acompanhamento a nivel ambulatorial, é recomendada a manutenção de drogas anticonvulsivantes pelo prazo de um ano, no caso de nāo haver alterações eletrencefalográficas ou queixas subjetivas. As alterações comportamentais costumam perdurar por prazos muito variáveis, na dependência do grau de sangramento e do local do An.

Um problema que ainda preocupa o profissional que acompanha um paciente com aneurisma refere-se à necessidade de seus familiares serem submetidos à investigação para a detecçāo do An antes do sangramento. A incidência em gêmeos univitelinos é extremamente alta. Algumas coletas de dados apontam como quatro anos o espaço de tempo médio para a ruptura de An no outro irmão gêmeo, após o primeiro sangramento em um deles. É interessante notar que a idade média do primeiro sangramento situa-se ao redor dos trinta e dois anos, portanto anterior à da população em geral. Com os novos métodos diagnósticos nãoinvasivos, muito em breve tal problema deverá ser resolvido. Os relatos de incidências familiares sĩo isolados e bastante restritos. As maiores proporções foram encontradas em uma população da Finlândia $(10 \%)^{23} \mathrm{e}$, mais recentemente, em Quebec, onde foram estudados parentes até de terceiro grau, com um indice de $32,2 \% \%^{+6,17.19 .23}$. A angiografia por cateterismo nos moldes atuais é considerada um exame bastante invasivo e não bem-aceito em pacientes assintomáticos, cujia única supeita reside em um grau de parentesco com paciente vítima de HSA.

O capítulo da HSA está longe de ser resolvido; muitos conhecimentos chegam diariamente em nossals mãos com mudanças fundamentais nas condutas e resultados. Acreditamos que a maior alteração ocorrerí quando for possível o diagnóstico do An em exame rotineiro e portanto, antes da sua ruptura, quando o tratamento tiver índices altíssimos de cura sem as sequielas da hemorragia.

\section{SUMMARY}

Spontaneous subarachnoid hemorrhage due to cerebral aneurisms

Clinical recovery after subarachnoid hemorrhage is still troublesome with high mortality rate. Aneurysm rupture accounts for about $80 \%$ of spontaneous subarachnoid hemorrhage in adults and $55 \%$ in children. Rebleeding and cerebral vosospasm are the main causes of morbidity and mortality. Some new drugs have been developed to counteract vasospasm. Mild hypothermia has proved to be the best protective agent so far. New surgical approaches, techniques and modern clips have improved the outcome after spontaneous subarachnoid hemorhage. Some general topics are discussed.

\section{KEYWORDS}

Subarachnoid hemorrhage; cerebral hemorrhage; brain aneurysms

\section{Referências}

1. Astrup, J.; Siesjo, B. K.; Symon, L. Thresholds in cerebral ischemia penumbra. Stroke, 12: 723.725, 1981.

2. Aymard, A.; Gobin, Y. P.; Casarco, A.; George, B.; Rousseau, P.; Merland, J.J. Aneurysmes intra-crâniens multiples: Traitment endovasculaire pour coils. Neurochirurgie, 38: 353 . 357, 1992.

3. Bolger, C.; Phillips, J.; Gilligan, S.; Tarek, Z.; Michael, F.; Croake, D.; Bouchier, D. Elevated levels of Lipoprotein a in association with cerebrovascular saccular aneurysm disease. Neurosurgery, 37: 241-246, 1995.

4. Brisman, R.; Abassioun, K. Familial intracranial aneurysm. J Neurosurg, 34: 678-682, 1971

5. Butter, H.; Peterson, J.W.; Zervas, N.T.; Morgan, K.G. Intracellular calcium, myosin light chain phosphorylation and contractile force in experimental cerebral vasospasm. Neurosurgery, 38: 781-787, 1996. 
6. Butler, W.E.; Barker, F.G.; Crowell, R.M. Patients with polycystic kidney disease would benefit from routine magnetic resonance angiografic screening for Intracerebral aneurysms: a decision analysis. Neurosurgery, 38: 506-515, 1996.

7. Coimbra, C.; Wieloch, T. Moderate hypotermia mitigates neuronal damage in the rat brain when initiated several hours following transient cerebral ischemia. Acta Neuropathol, 87: 325-331, 1994.

8. Coimbra, C.; Carvalho, A.C.; Oliveira, R.J.; Sinigaglia, R.; Santos, G.; Cavalheiro, E.A. Effects of reperfusion under moderate hypothermia on ischemic brain damage. Ciència $e$ Cultura, 47: 266-268, 1995.

9. Crawford, M.D.; Sarner, M. Ruptured intracranial aneurysms. Lancet, 52: 1254-1257, 1965.

10. Fendlay, M.; Kassel, N.; Weir, B.; Haley, C.E.; Kongable, G.; Germanson, T.; Truhowsky, L.; Alves, W.; Yonas, H.; Stemberg, G.; Winn, R.; Ferguson, G. A randomized trial of intraoperative intracisternal tissue plasminogen activator for the prevention of vasospasm. Neurosurgery, 37: 241-249, 1995.

11. Graves, J.B.; Strother, C.M.; Thomas, A.; Perl, J. Early treatment of ruptured aneurysm with Guglielmi detachable coil:effect on subsequent bleeding. Neurosurgery, 37:640. $644,1995$.

12. Guglielmi, G.; Viñuela, F.; Ducwiler, G.; Dion, J. Electrothrombosis of saccular aneurysms via endovascular approach. J Neurosug, 75: 515-524, 1992.

13. Hsiang, J.N.K.; Liang, E.Y.; Lam, J.M.K.; Zhu, X.L.; Poon, W.S. The role of computed tomographic angiography in the diagnosis of intracranial aneurysms and emergent aneurysm clipping. Neurosurgery, 38: 481-487, 1996.

14. Hunt, W.E.; Hess, R.M. Surgical risk as related to time of intervention in the repair of intracranial aneurysms. J Neurosurg, 28: 14-20, 1968.

15. King, J. ; Berli, A.; Flamm, E.S. Morbidity and mortality from elective surgery for assymptomatic unruptured intracranial aneurysms: a meta-analisys. J Neurosurg, 81:837-842, 1994.

16. Lawton, M.; Ho, J.C.; Bichard, W.D.; Coons, S.W.; Zabramski, J.M.; Spetzler, R.F. Titanium aneurysm clip: part I: mechanical, radiological and biocompactibility testing. Neurosurgery, 38: 1158-1164, 1996.

17. Lozano, A.; Leblanc, R. Familial intracranial aneurysms. J Neurosurg, 66: 522-528, 1987.
18. Markarian, G.Z.; Lee, J.H.; Stein, D.J.; Hong, S.C. Mild Hypotermia: therapeutic window after cerbral ischemia. Neurosurgery, 38: 542-551, 1996.

19. O'Brian, J.G. Subarachnoid hemorrhage in identical twins. Br Med J, 1: 607-609, 1942.

20. Ogilvy, C.; Chu, D.; Kaplan, S. Mild hypothermia, hypertension and mannitol are protective against infarction during experimental intracranial temporary vessel oclusion. Neurosurgery, 38: 1202-1210, 1996.

21. Ohta, T.; Kuroiwa, T.; Sakaguchi, I.; Sakai, N.; Moriwaki, K. Selective hypothermic perfusion of canine brain. Neurosurgery, 38: 1211-1215, 1996.

22. Rest, M.; Cruthman, R.D. Absence of collagen deficiency in familial cerebral aneurysms. J. Neurosurg, 70: 837-840, 1989.

23. Ronkainen, A.: Heinesneim, J. Familial subarachnoid hemorrhage in Finland. Neurosurgery, 37: 787-797, 1993.

24. Sekar, L.N.; Kaliak, K.; Yonas, H.; Wright, D.C.; Ching, H.; Heros, R.C.; Batjer, H.H.; Smith, R.R. Cranial base approaches to intracranial aneurysm in the subarachnoid space. Neurosurgery, 35: 472.483, 1994.

25. Sinar, E.J.; Mendelow, A.D.; Grahan, D.J.; Teasdale, M.G. Experimental intracerebral hemorrhage: effects of a temporary mass lesion. J Neurosurg, 66: 568-576, 1987.

26. Tymianski, M.; Tator, C. Normal and abnormal calcium homeostasis in neurons: a basis for the pathophysiology of traumatic and ischemic central nervous system injury. Neurosurgery, 38: 1176-1195, 1996.

27. Wheelock, B.; Wier, B.; Watts, R.; Mohr, G.; Khan, M.; Fewer, D.; Ferguson, C.; Durity, F.; Cochrane, D.; Benoit, B. Timing of surgery for intracranial hematomas due to aneurysm rupture. J Neurosurg, 58: 476.481, 1983.

28. Yamamoto, S.; Golonov, E.V.; Berger, S.B.; Reis, D.F. Inhibition of Nitric Oxid syntesis increases focal ischemic infarction in rat. J Cereb Blood Flow Met, 12: 717-726, 1992.

\section{Endereço para correspondência:}

Mirto N. Prandini

Rua dos Crisântemos, 117

04049-020 Săo Paulo (SP) 